\section{Influencia de los factores socioculturales en el Trastorno de Conducta Alimentaria}

\section{Influence of sociocultural factors in Eating Disorders}

\section{Sr. Editor}

En un artículo previo realizado por Behar et al., se indica la importancia de la relación de la insatisfacción corporal (IC) y del perfeccionismo (P) como factores predisponentes para desarrollar trastornos de conducta alimentaria $(\mathrm{TCA})^{1}$. Asimismo, el artículo destaca la importancia del estudio de los factores socioculturales vinculados con el incremento de la IC y, consecuentemente, de los TCA ${ }^{2}$.

Actualmente, el aumento de TCA en la población joven está influenciada por la cultura de la delgadez y se busca explicar a través de modelos multifactoriales. Uno de los más importantes es el "Tripartite Influence Model", el cual propone tres fuentes básicas primarias de presión: padres, pares y medios de comunicación ${ }^{2,3}$. En el artículo de Behar et al., se menciona el modelo tripartito así como diversas escalas para medir la IC que son relevantes para el estudio de los TCA.

Existen diversas escalas para la evaluación de las presiones del modelo tripartito y cada una de éstas mide una presión diferente ${ }^{2}$. Sin embargo, en 2014 Llorente et al., validaron la versión española del Sociocultural AttitudesToward Appearance Ques- tionnaire-4 (SATAQ-4), el cual posee 22 ítems en los que se emplea una escala de tipo Likert que va desde "completamente en desacuerdo" a "completamente de acuerdo". Estos ítems se encuentran divididos en 5 dominios dentro de los que se incluyen y evalúan las tres presiones del modelo tripartito (Tabla 1). El SATAQ-4 ha mostrado una alta consistencia interna en población universitaria, así como un alfa de Cronbach para la escala global de $0,93^{4}$.

La primera versión del Sociocultural AttitudesToward Appearance Questionnaire (SATAQ-1) fue plasmada en 1994 con el ideal de estudiar tanto la influencia sociocultural sobre la imagen corporal como la insatisfacción corporal en jóvenes y adolescentes. Desde el inicio, esta escala mostró la interiorización que las presiones sociales ejercían sobre el sentido de apariencia en mujeres jóvenes ${ }^{5}$. Dada la relevancia de este primer estudio y la replicación del mismo en otros países, se fueron creando versiones actualizadas del mismo en busca de la mejora del instrumento. En 2003, Smith et al., validaron la versión 3 del Sociocultural Attitudes Toward Appearance Questionnaire (SATAQ-3), la cual evaluaba de manera eficaz la influencia que ejercían los medios de comunicación sobre el ideal de delgadez, más no las otras presiones propuestas en el modelo tripartito ${ }^{6}$. Es por tal motivo que se creó la nueva versión mencionada anteriormente, en busca de un mejor estudio de los factores socioculturales que influyen en el desarrollo de IC y TCA $^{4}$.

En base a lo expuesto, consideramos Sr. Editor que es de interés mencionar la relevancia del SA-

Tabla 1. Sub-escalas del SATAQ-4

\begin{tabular}{|lll|}
\hline Sub-escalas del SATAQ-4 & Alfa de Cronbach & 0,90 \\
Sub-escalas de internalización & Internalización de la ideal delgadez & 0,89 \\
& Internalización del ideal atlético & 0,88 \\
Sub-escalas de presión & Presión por parte de los padres & 0,94 \\
& Presión por parte de los medios de comunicación & 0,97 \\
\hline
\end{tabular}

Elaboración basada en datos publicados en Translation and Validation of Spanish Version of the Socio cultural Attitudes Towards Appearance Questionnaire-4 (SATAQ-4). 
TAQ-4 como una escala para la medición de las presiones ambientales según el modelo tripartito, así como también la utilidad de su aplicación con fines de estudio en la población latinoamericana.

Carolina Zevallos-Delzo ${ }^{1}$, Manuel M. Catacora-Villasante ${ }^{2,3}$

${ }^{1}$ Estudiante, Escuela de Medicina, Universidad Peruana de Ciencias Aplicadas, Lima, Perú. ${ }^{2}$ Médico Psiquiatra, Hospital Hermilio Valdizán. ${ }^{3}$ Docente, Escuela de Medicina, Universidad Peruana de Ciencias Aplicadas, Lima, Perú.

\section{Referencias bibliográficas}

1. Behar R, Gramegna G, Arancibia M. Perfeccionismo e insatisfacción corporal en los trastornos de la conducta alimentaria. Rev Chil Neuro-Psiquiatr 2014; 52 (2): 103-14.

2. Van den Berg P, Thompson J. K, ObremskiBrandon K, Coovert M. The Tripartite Influence model of body image and eating disturbance A covariance structure modeling investigation testing the meditational role of appearance comparison. J Psychosom Res 2002; 53: 1007-20.

3. Grogan S. Body Image, understanding body dissatisfaction in men, woman and children. 2nd ed. New York: Routledge; 2008.

4. Llorente E, Gleaves D, Warren C, Pérez L, Rakhkovskaya L. Translation and Validation of a Spanish Version of the Sociocultural Attitudes Towards Appearance Questionnaire-4 (SATAQ-4). Int J Eat Disord. 2014. [Epub ahead of print].

5. Heinberg L, Thompson K, Stormer S. Development and Validation of the Sociocultural Attitudes Towards Appearance Questionnaire. Int J of Eat Disor 1995; 17 (1): 81-9.

6. Thompson K, van den Berg P, Roehrig M, Guarda A, Heinberg L. The Sociocultural Attitudes Towards Appearance Scale-3 (SATAQ-3): Development and Validation. Int J Eat Disord 2004; 35 (3): 293-304.

Correspondencia:

Carolina Mercedes Zevallos Delzo

Calle Coronel Inclán 553 interior 604, Miraflores.

Teléfono: +0514454449

E-mail:cmzdelzo@gmail.com 